\title{
Nurses Can Reduce the Patient's Length of Stay in Emergency Department: An Quality Initiatives
}

\author{
Naseem Akhtar ${ }^{*}$, Awais Jamil ${ }^{2}$, Sana Sehar ${ }^{3}$, Muhammad Afzal ${ }^{4}$, Dr. Syed Amir Gilani ${ }^{5}$ \\ ${ }^{1}$ BSN (Student), Lahore School of Nursing the University of Lahore (New Campus), Lahore, Punjab, Pakistan \\ ${ }^{2}$ Clinical Nurse Manager, Tertiary Care Hospital Karachi, Pakistan \\ ${ }^{3}$ Assistant Professor at LSN (UOL), Lahore School of Nursing the University of Lahore (New Campus), Lahore, Punjab, Pakistan \\ ${ }^{4}$ Professor at LSN (UOL), Lahore School of Nursing the University of Lahore (New Campus), Lahore, Punjab, Pakistan \\ ${ }^{5}$ Dean: Faculty of Allied Health Sciences, Lahore School of Nursing the University of Lahore (New Campus), Lahore, Punjab, Pakistan
}

DOI: $10.36348 /$ sjnhc.2019.v02i12.002

| Received: 20.11.2019 | Accepted: 27.11.2019 | Published: 18.12.2019

*Corresponding author: Naseem Akhtar

\section{Abstract}

Introduction: The mean of patient's length of stay in the Emergency Department is considered to manage the patient's crowing. This paper measures the length of stay and factors that could potentially influence on patient's stay in emergency department. Objective(s): To reduce length of stay and early disposition of patient in in patient area for continuity of care. Design: Prospective study design used and evaluate intervention to improve patient safety. Settings: Emergency Department of a Tertiary Hospital Lahore Pakistan Methodology: By using the change theory of management we identify the factors which impact on Length of stay Educate and motivate staff, we developed and implemented Nurse physician hand over checklist as well as developed LOS tool in emergency, furthermore staff reviewed patient and gave reminder to consultant a fro early bed vacation. Moreover we empowered our nursing staff. Results: After implementation of kut Lewis model, there is huge fluctuation in the graph through march 2018 to January 2019 and it significantly increased in the month of February 2019 and the emergency length of stay dropped fro $\mathrm{m} 4.1$ to 5 hours after we reviewed our process, retrained staff in the month of February after training and followup results dropped dramatically to 3 hours by March until April 2019. Conclusion: In conclusion, all factors directly impact on patient length of stay for this purpose management developed some strategies against this to overcome this issue. These strategies really help especially the bed management team played very important role on it.

Keywords: Length of stay, Patient, Emergency department, Crowd.

Copyright @ 2019: This is an open-access article distributed under the terms of the Creative Commons Attribution license which permits unrestricted use, distribution, and reproduction in any medium for non-commercial use (NonCommercial, or CC-BY-NC) provided the original author and source are credited.

\section{INTRODUCTION}

Emergency Deoartment length of stay is define $\mathrm{d}$ as the interval between Emergency Ddepartment time of arrival and ED time of departure (either admission or discharge) [1]. Emergency department (ED) mean length of stay (LOS) per patient measured from arrival to departure was promoted as a surrogate crowding indicator in the absence of a standard or universal definition [2].

Benchmarking is a comparison and measurement of the services provided by a healthcare organization to other national healthcare organizations. It provides insight to leaders to help them understand how they compare their organization with similar organizations providing the same services [3]. Because of the persistent increase in inpatient volumes of emergency departments, it has become more important from an operational point of view to improve the timeliness of emergency care delivery. Time-based variables and the scheduling of staffing may increase the timeliness of delivery of emergency care [4].

Common influential factors such as age, patient entity, acuity level of triage, or time of arrival may have different effects on different patient disposition groups [4]. Length of stay (LOS) is considered a key measure of emergency department utilization, and from the point of view of the patient, it is viewed as a measure of healthcare service quality. High LOS can lead to increases in costs and may also have consequences for patient safety, while other organizational improvements, communication enhancement, and time management may have a positive effect on it.

Length of stay (LOS) is considered a key measure of emergency department utilization, and from 
the point of view of the healthcare provider, it is considered as a measure of healthcare service quality [6].

\section{BACKGROUND}

It had been observed a plethora of variations in the Emergency length of staying our targeted LOS is < 4 hours which we achieved in Jan 2018 which was 3.1 but after that, it's increased drastically and touched to the nadir in the month of March 2018 which was 5.02 there were ample of reason to increased LOS.

1. The emergency was not designed properly.

2. Bed occupancy issues in inpatient areas.

3. Communication Issues.

4. Issues in handed over.

5. No buddy took ownership in an emergency.

6. The conflict between doctors and nurses.

7. No, any checklist develop in the EAR

8. No, any hand over sheet to develop for handed over.

According to Mercer, Singh, and Kanzaria explained in 2019 that Emergency is the critical area and overcapacity is one of the major issues which we are facing in our setup [7]. Regardless of an extensive belief that low hospital bed availability we try to accommodate patients and overcome the crowding issue in Emergency, secondly we were keenly observed the impact of the early disposition of patients in the inpatient area will help to reduce the EAR LOS.

Emergency length of stay is one the quality indicator of our department so that why we reevaluate our process and find out the cause of the delayed inpatient care and length of stay as well.

The aim of this study is to reduce the length of stay and early disposition of patient inpatient area for continuity of care.

\section{MANAGEMENT THEORY}

The decision making style have a transformational leadership. In the transformational model, leader works on enabling and improvement of their subordinates. They decorate a future picture in front of their followers and striving towards the mission. Generally transformational leader improves the satisfaction level of employees and patient as well as outcome [8].

Health care organizational have a great changes in restructuring and modifications which are increasingly have complex chaos and problems in different situation. Transformational leadership style helps to solve the issue [9].

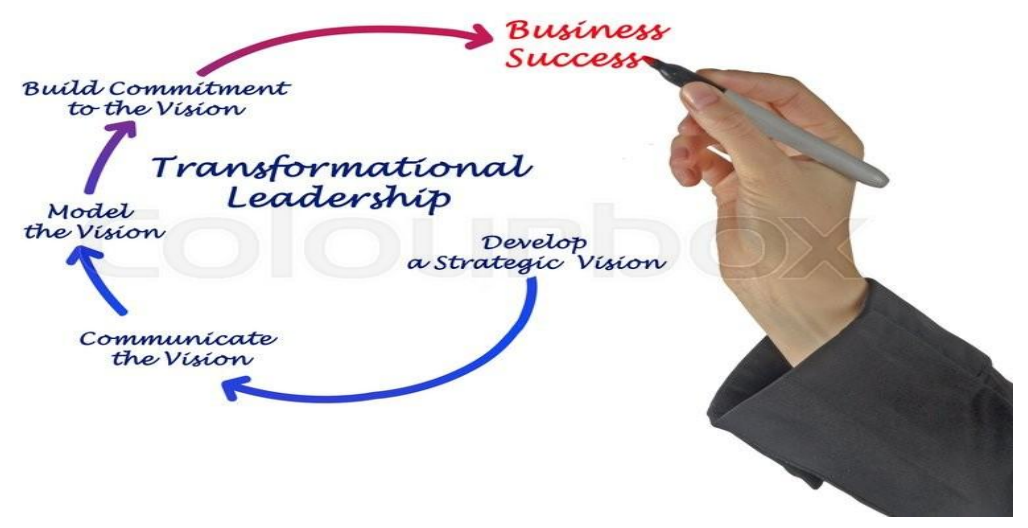

\section{RATIONAL}

According to scenario, transformational leadership theory and decision making approach can be applied in this situation as command to staff do fast process for reducing the length of stay, work in collaboration with other medical team, take the thought of team about solving the issue and work with strategies plan to improvement and expected output.

In this review, we collected data retrospectively from tertiary care cancer hospital and we reviewed patient daily census, admission rate, occupancy rate meanwhile we observed how many patients direct discharge from EAR, number of patients stay in an emergency, till admission request and LOS for admission request to bed.

\section{APPLICATION OF CHANGE THEORY}

This was an observational study design using Emergency data through electronic medical record and only the emergency population enrolled in this study. The Emergency consist of 13 beds 8 in adult and 6 in pages. All patients presenting to the Emergency between January 2018 and September 2019 were included in the study. The predictor variable was daily hospital occupancy. Outcome measures included the daily emergency length of stay for admitted patients and the daily admission rate. Apply change theory model in our study.

In the unfreezing phase, we study the different cause which effects on length of stay by using the fish 


\section{CAUSE \& EFFECTS DIAGRAM}

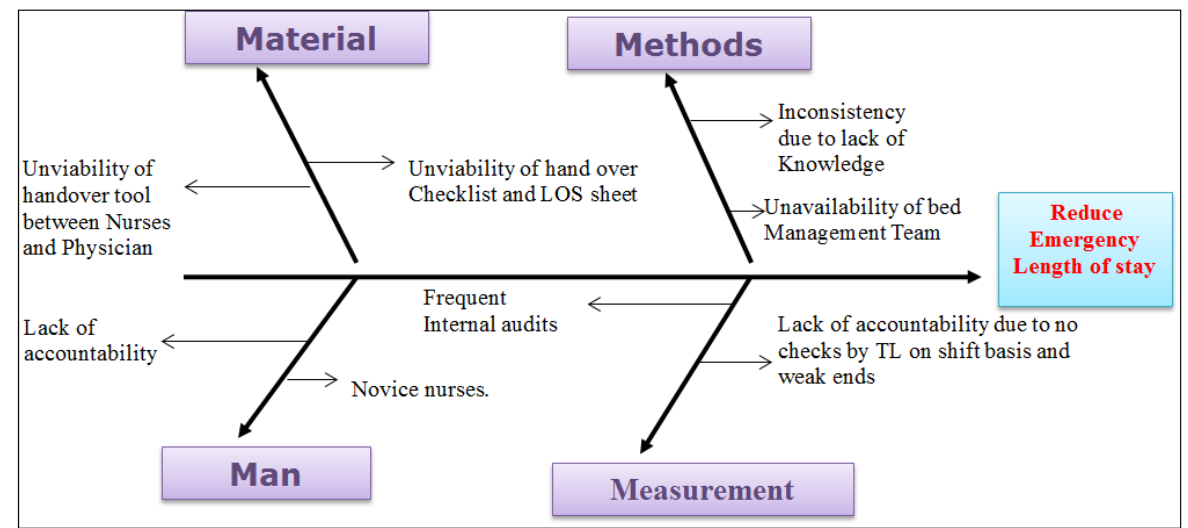

In the moving stage, We developed an implementation plan we started with need assessment and identified the different factors which impact on length of stay we discussed a plan with team and started brainstorming that how we overcome that issues in future Also shred our plan with staff in morning meetings and our team developed a checklist for LOS and also we initiated that nurse-physician hand over sheet through these sheet nurses handed overall patients to physician at the end of the in addition nurses give reminder called to physician that patient LOS increased in EAR so reviewed that patients.

\begin{tabular}{|l|l|l|l|l|l|l|}
\hline \multicolumn{7}{|c|}{ Emergency Assessment Room Reason for increased length of stay } \\
\hline Sr. No & Patient Name & MR \# & Date \& Time & Central Nurse & Reason for Delay & $\begin{array}{l}\text { Consultant Comments } \\
\text { Acknowledgement }\end{array}$ \\
\hline 1 & & & & & & \\
\hline 2 & & & & & & \\
\hline 3 & & & & & & \\
\hline 4 & & & & & & \\
\hline
\end{tabular}

This initiative builds nurses' confidence level as well as nursing staff-owned that responsibility moreover we presented our data on weekly basis in the EAR core group meeting as well as shared that data with the medical director on monthly basis. Secondly, we also shared data with nursing staff in the morning meeting and take sessions on it that we filled those gaps in the future. We motivated our staff and started monthly appreciation with a direct effect on staff performance. The nursing division initiated bed management team this team conducted the meeting on daily basis and they discussed how many patients waiting in emergency and admission holding bay and further they coordinated with the department furthermore at the end of the shift the coordinate with the heads and submit a daily report to the nursing division this activity really helpful.

Additionally, we learned changed theory and we implemented that model in our practice.

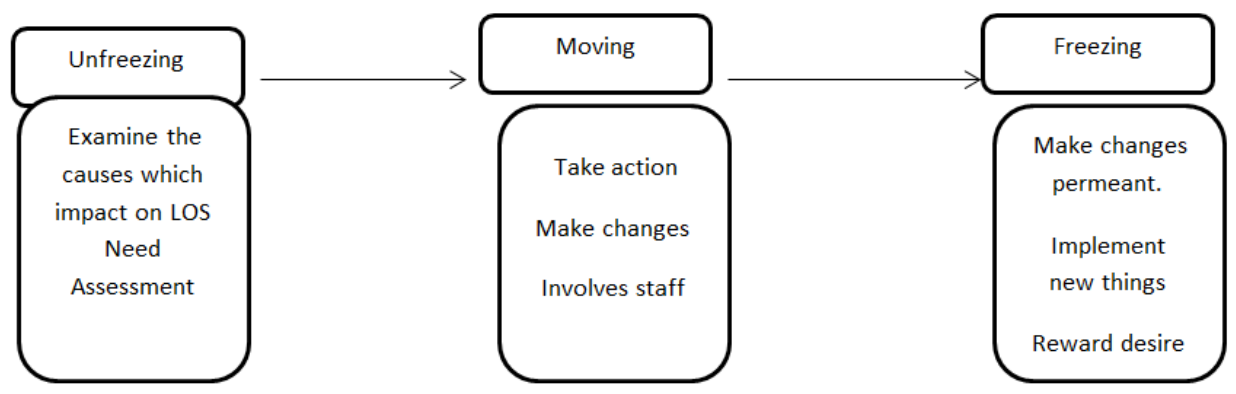

(Mitchell, 2003) [10]

\section{RESULTS}

After Implementation there is immense of fluctuation in the graph through March 2018 till Jan 2019 and it significantly increased in the month of February 2019 and the emergency length of stay inclined 4.1 to 5 hours after that we reviewed our process, reeducate our staff in the month of Feb after education and follow-up the results dramatically decreased through march till April 2019, 5hours to 3.3 hours and its drastically improved in the month of May 


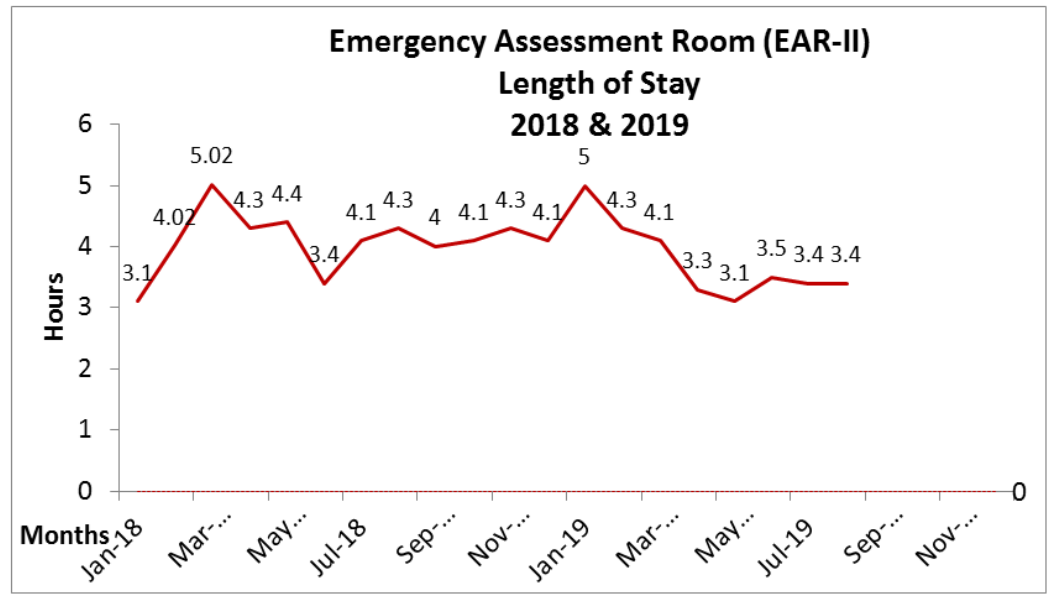

\section{DISCUSSION}

The team starts brainstorming what was the contributing factor that increased Length of stay this concept led to innovative ideas after that we search for literature from the year 2014 to 2019. 20 were reviewed number article search from the database. CINAHIL, PUBMED, GOOGLE SCHOLAR, 10 abstract only 5 have the recommendation to reduce LOS in an emergency, most of the studies were recommended the same thing mentioned above. One of the studies strongly recommended developing a protocol or Algorithm for emergency LOS and teaching staff according to Algorithm [11].

According to Tenbensel et al., [12], many researchers suggested that multiple rounds, feedback, and education can help to reduce the length of stay significantly with the support of leadership and strategies to improve patient flow. We adopted the different strategies and practices to improve the communication between physician, laboratory and radiology.

An appreciation and motivation help staff to do their work timely and with passion and responsibility. In our scenario, appreciation increased the result of reducing patient's length of stay. However, Nurses work in a healthcare context in which they have to merge cost-effectiveness and accountability with their desire to provide patient-based care and preferencesbased nursing care, and they experience a conflict between these two approaches [13].

In the future, I suggested to develop a policy, protocol, and Algorithm and will teach staff accordingly. Moreover, the organization develops a bed management team that is responsible for bed allocation, early discharges, and early disposition of patient emergency to the inpatient Area.

\section{CONCLUSION}

In nutshell, the all contributing factors increased the length of stay so the checklist or tool will really help to reduce LOS secondly the bed Management team has played an important role in it. Moreover, the nurses should be empowered for early bed vacation really helps to reduce the emergency length of stay.

\section{REFERENCE}

1. Fee, C., Burstin, H., Maselli, J. H., \& Hsia, R. Y. (2012). Association of emergency department length of stay with safety-net status. Jama, 307(5), 476-482.

2. Rathlev, N. K., Obendorfer, D., White, L. F., Rebholz, C., Magauran, B., Baker, W., ... \& Olshaker, J. (2012). Time series analysis of emergency department length of stay per 8-hour shift. Western Journal of Emergency Medicine, 13(2), 163.

3. Alvin, C. (2019). Murfreesboro, \& Tenn. Clinical Nurse Educator. York VA Medical Center. Retrieved from [November 28, 2019]. https://journals.lww.com/nursingmadeincrediblyea sy/Fulltext/2015/03000/Let_s_talk_about_benchm arking.1.aspx

4. Sarıyer, G., Ataman, M. G., \& Kızıloğlu, İ. (2018). Factors affecting length of stay in the emergency department: A research from an operational viewpoint. International Journal of Healthcare Management, 1-10.

5. Chaou, C. H., Chiu, T. F., Yen, A. M. F., Ng, C. J., $\&$ Chen, H. H. (2016). Analyzing factors affecting emergency department length of stay-using a competing risk-accelerated failure time model. Medicine, 95(14).

6. Bashkin, O., Caspi, S., Haligoa, R., Mizrahi, S., \& Stalnikowicz, R. (2015). Organizational factors affecting length of stay in the emergency department: initial observational study. Israel journal of health policy research, 4(1), 38.

7. Mercer, M. P., Singh, M. K., \& Kanzaria, H. K. (2019). Reducing Emergency Department Length of Stay. JAMA, 321(14), 1402. 
8. Swanwick, T., \& McKimm, J. (2017). ABC of Clinical Leadership. Hoboken, NJ: John Wiley \& Sons.

9. Roussel, L. A., $\quad$ Swansburg, R. C., \& Swansburg, R. J. (2006). Management and Leadership for Nurse Administrators. Burlington, MA: Jones \& Bartlett Learning.

10. Mitchell, G. (2013). Selecting the best theory to implement planned change. Nursing Management, 20(1), 32-37.

11. Vahdat, V., Griffin, J., \& Stahl, J. E. (2017). Decreasing patient length of stay via new flexible exam room allocation policies in ambulatory care clinics. Health Care Management Science, 21(4), 492-516.

12. Tenbensel, T., Chalmers, L., Jones, P., AppletonDyer, S., Walton, L., \& Ameratunga, S. (2017). New Zealand's emergency department target-did it reduce ED length of stay, and if so, how and when?. BMC health services research, 17(1), 678.

13. Kieft, R. A., de Brouwer, B. B., Francke, A. L., \& Delnoij, D. M. (2014). How nurses and their work environment affect patient experiences of the quality of care: a qualitative study. BMC health services research, 14(1), 249. 